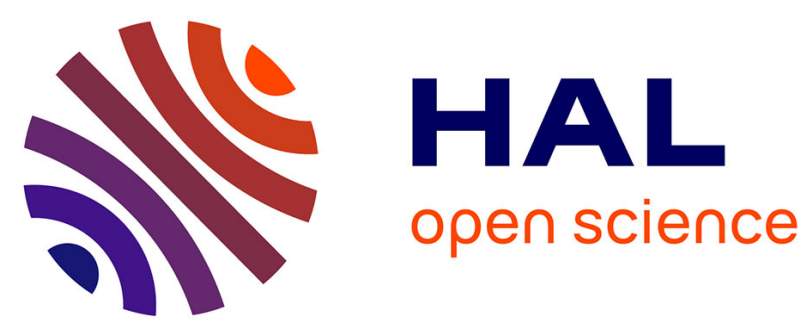

\title{
Observability of laminar bidimensional fluid flows seen as autonomous chaotic systems
}

Gisela D Charó, Denisse Sciamarella, Sylvain Mangiarotti, Guillermo Artana, Christophe Letellier

\section{- To cite this version:}

Gisela D Charó, Denisse Sciamarella, Sylvain Mangiarotti, Guillermo Artana, Christophe Letellier. Observability of laminar bidimensional fluid flows seen as autonomous chaotic systems. Chaos: An Interdisciplinary Journal of Nonlinear Science, 2019, 29 (12), pp.123126. 10.1063/1.5120625 . hal03029728

\section{HAL Id: hal-03029728 \\ https://hal.science/hal-03029728}

Submitted on 28 Nov 2020

HAL is a multi-disciplinary open access archive for the deposit and dissemination of scientific research documents, whether they are published or not. The documents may come from teaching and research institutions in France or abroad, or from public or private research centers.
L'archive ouverte pluridisciplinaire HAL, est destinée au dépôt et à la diffusion de documents scientifiques de niveau recherche, publiés ou non, émanant des établissements d'enseignement et de recherche français ou étrangers, des laboratoires publics ou privés. 


\section{Observability of laminar bidimensional fluid flows seen as autonomous chaotic systems}

Cite as: Chaos 29, 123126 (2019); https://doi.org/10.1063/1.5120625

Submitted: 19 July 2019 . Accepted: 02 December 2019 . Published Online: 18 December 2019

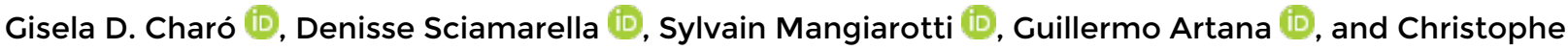
Letellier (iD)

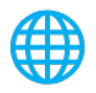

\section{AIP Conference Proceedings}

FLASH WINTER SALE!

$50 \%$ OFF ALL PRINT PROCEEDINGS 


\title{
Observability of laminar bidimensional fluid flows seen as autonomous chaotic systems
}

\author{
Cite as: Chaos 29, 123126 (2019); doi: 10.1063/1.5120625 \\ Submitted: 19 July 2019. Accepted: 2 December 2019 \\ Published Online: 18 December 2019

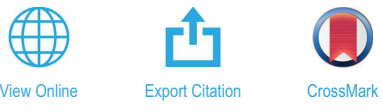

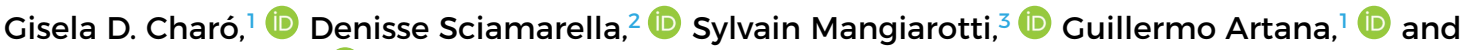
Christophe Letellier ${ }^{4}$ (i)

\author{
AFFILIATIONS \\ ${ }^{1}$ Laboratorio de Fluidodinámica, Facultad de Ingeniería, Universidad de Buenos Aires, CONICET, C1063ACV CABA, Argentina \\ ${ }^{2}$ Institut Franco-Argentin d'Études sur le Climat et ses Impacts (IFAECI), UMI 3351 (CNRS-CONICET-UBA), \\ C1428EGA CABA, Argentina \\ ${ }^{3}$ Centre d'Études Spatiales de la Biosphère, UPS-CNRS-CNES-IRD, Observatoire Midi-Pyrénées, 18 avenue Édouard Belin, 31401 \\ Toulouse, France \\ ${ }^{4}$ Normandie Université_CORIA, Campus Universitaire du Madrillet, F-76800 Saint-Etienne du Rouvray, France
}

\begin{abstract}
Lagrangian transport in the dynamical systems approach has so far been investigated disregarding the connection between the whole state space and the concept of observability. Key issues such as the definitions of Lagrangian and chaotic mixing are revisited under this light, establishing the importance of rewriting nonautonomous flow systems derived from a stream function in autonomous form, and of not restricting the characterization of their dynamics in subspaces. The observability of Lagrangian chaos from a reduced set of measurements is illustrated with two canonical examples: the Lorenz system derived as a low-dimensional truncation of the Rayleigh-Bénard convection equations and the driven double-gyre system introduced as a kinematic model of configurations observed in the ocean. A symmetrized version of the driven double-gyre model is proposed.
\end{abstract}

Published under license by AIP Publishing. https://doi.org/10.1063/1.5120625

The motion of fluid is involved in many natural situations such as in the atmosphere, oceans, stars where the fluid is charged, many industrial processes such as in cooler and mixing tanks, and even in physiology with the blood circulation and ventilation. Understanding the motion of fluid particles is, therefore, of primary importance. A variety of mixing and transport problems involving incompressible bidimensional flows has been undertaken using a nonlinear dynamics approach, overlooking embedology and observability issues that are discussed for the first time in this work. When a stream function is written, an analogy between conservative flow and bidimensional incompressible flow can be exhibited. Interesting features such as chaotic mixing and nonmixing islands are commonly observed. It is shown here that such flows should be investigated in the state space and not only in the physical space, which is a plane projection of the higherdimensional state space. The advantages of working in the whole state space are illustrated with two paradigmatic examples.

\section{INTRODUCTION}

The Eulerian and Lagrangian descriptions of the dynamics of a fluid can be very different. ${ }^{1}$ In the Eulerian description, the system under consideration is a control volume of fluid flow. In this formulation, the space-time coordinates, which reduce to $(x, z, t)$ in a two-dimensional fluid flow, are used as independent variables. In the Lagrangian approach, the system under consideration governs the motion of the fluid particles; the spatial coordinates $(x, z)$ are dependent variables and $t$ remains the sole independent variable, as temporal systems are considered in the dynamical systems theory. The main difference between the Eulerian and the Lagrangian descriptions is that the $x$ - and $z$-coordinates have a different status.

The connection between Lagrangian transport and the dynamical systems theory is stressed when the flow is not only twodimensional but also incompressible. ${ }^{2}$ In this case, it is possible to express the velocity field in terms of a scalar field, leading to a stream function $\Psi$. The equations governing the motion of fluid particles thus take the form of a Hamiltonian system, the stream function playing a role similar to the role of a Hamiltonian function..$^{2-4}$ Some dynamical properties observed in conservative systems can also be observed in incompressible two-dimensional flows and the Kolmogórov-Arnold-Moser (KAM) theory can be used to understand their dynamics. ${ }^{2-4}$

Usually, the velocity field is time-dependent and so are the equations governing the motion of fluid particles. The time-dependence 
of the velocity field is generally governed by partial differential equations, that is, by an infinite-dimensional dynamics. It may be reduced to a few modes by using a Galerkin technique. ${ }^{4}$ Rather than explicitly writing the equations governing the dynamics, these modes may be injected as a driving term in the equations governing the motion of fluid particles. In other words, the dynamical system associated with the motion of fluid particles is nonautonomous, for which the state space remains not explicit. ${ }^{5}$ The advantage of rewriting a nonautonomous system in an autonomous form is that the classical tools developed in the context of the nonlinear dynamical systems theory can be applied without any restriction. We will show that such autonomous writing helps clarifying concepts as Lagrangian chaos and chaotic mixing.

According to the nonlinear dynamical systems theory, the dynamics produced by a system must be ideally investigated in the state space, that is, in the space spanned by all the variables involved in the governing equations. In practical situations, all these variables are only rarely measured, and it is necessary to investigate the dynamics from a reduced set of measured variables. This was addressed in the case of geosciences ${ }^{6}$ and the use of derivatives of the measured time can allow reconstruct the missing information. In particular, determining an optimal set of reduced measurements is important when data assimilation must be used to recover the needed information. ${ }^{8,9}$ It is possible to check that all the required information for distinguishing every state of the system is indeed contained in the measurements by means of the observability matrix, ${ }^{10}$ that is, the Jacobian matrix of change of coordinates between the original state space and the space reconstructed from the derivatives of the measured variables. ${ }^{11}$ There is full observability of the original space when there is no loss of information in the measurements. An observability analysis may, therefore, ensure that all the properties of the dynamics are actually "seen" by the measurements. This will help us to check whether the variables related to the Lagrangian description can provide a reliable characterization of the dynamics or not.

Two benchmark models for two-dimensional incompressible fluids will be used to illustrate the importance of rewriting the systems in an autonomous form and of performing an observability analysis: the truncated solutions of the Rayleigh-Bénard (RB) convection problem ${ }^{12}$ whose velocity field evolves according to the 1963 Lorenz system, ${ }^{13}$ and the driven double-gyre (DG) equations introduced as a kinematic model inspired in the ocean gyres. ${ }^{14-17}$ Both models are defined in terms of a stream function. The Lorenz system belongs to the paradigm of chaos. The driven double-gyre system has a long history as a simplified Lagrangian transport model ${ }^{18}$ proved to produce chaotic behavior. ${ }^{19}$ It is among the prototype models that are customarily used for testing Lagrangian coherent structure detection techniques. ${ }^{18,20-23}$

The subsequent part of this paper is organized as follows. Section II introduces the definitions of Eulerian and Lagrangian chaos when the system is rewritten in an autonomous form. Section III is devoted to a Rayleigh-Bénard convection in the context of a truncation of a Galerkin decomposition. The driven doublegyre flow is investigated in Sec. IV where it is shown that it can be written as a four-dimensional autonomous conservative system. A symmetrized and further simplified driven double-gyre model is also proposed in which the symmetry between two adjacent cells is preserved. Section $V$ gives some concluding remarks. An Appendix with a brief introduction to observability is provided.

\section{EULERIAN AND LAGRANGIAN FORMALISMS AND STREAM FUNCTIONS}

When a dynamical system is governed by a set of equations where the time explicitly occurs, the system is said to be nonautonomous. This means that some processes involved in the dynamics are not explicitly described and that the state space is not completely determined. Working in a space whose dimension is increased by one setting $\dot{t}=1$ leads to some difficulties for using the tools borrowed from the nonlinear dynamical systems theory-for instance, the state space is no longer bounded. It is thus strongly recommended to rewrite the system in an autonomous form to overcome them. This means that the equations describing the processes governing the driving term have to be inserted in the governing equations. ${ }^{5}$ These remarks are also valid for the nonautonomous differential equations

$$
\dot{x}=\boldsymbol{V}(\boldsymbol{x}, t)
$$

governing the motion of a fluid particle initially located at $x(0)$. When the velocity field is time-dependent, a Galerkin method can be applied and $m$-modes be retained for constructing an approximation. The time-dependence of the velocity field is described by the dynamical system,

$$
\dot{\gamma}=f(\gamma)
$$

where $\gamma$ is the vector of $m$-modes retained in the truncation. Let us design by $\mathscr{V}=\mathscr{V}(t) \subset \mathbb{R}^{m}(\boldsymbol{\gamma})$ the solution to system (2).

Let us now consider a velocity field $\boldsymbol{V}=\boldsymbol{V}(x, z)$ where $x$ and $z$ are the variables spanning the physical space with $z$ the vertical axis. When the time-dependence of the velocity field is made explicit by $m$ Galerkin modes, the stream function $\Psi=\Psi(x, \gamma)$ fully determines the velocity field $\boldsymbol{V}(\boldsymbol{x}, \boldsymbol{\gamma})$ according to

$$
\mid \begin{aligned}
& V_{x}(\boldsymbol{x}, \boldsymbol{\gamma})=\frac{\partial \Psi(\boldsymbol{x}, \boldsymbol{\gamma})}{\partial z}, \\
& V_{z}(\boldsymbol{x}, \boldsymbol{\gamma})=-\frac{\partial \Psi(\boldsymbol{x}, \boldsymbol{\gamma})}{\partial x} .
\end{aligned}
$$

When the fluid is incompressible, the underlying dynamics is governed by the system

$$
\left\{\begin{array}{l}
\dot{x}=\left.\frac{\partial \Psi\left(x_{\mathrm{E}}, z_{\mathrm{E}}, \boldsymbol{\gamma}\right)}{\partial z_{\mathrm{E}}}\right|_{x_{\mathrm{E}}=x, z_{\mathrm{E}}=z}=V_{x}(\boldsymbol{x}, \boldsymbol{\gamma}), \\
\dot{z}=-\left.\frac{\partial \Psi\left(x_{\mathrm{E}}, z_{\mathrm{E}}, \boldsymbol{\gamma}\right)}{\partial x_{\mathrm{E}}}\right|_{x_{\mathrm{E}}=x, z_{\mathrm{E}}=z}=V_{z}(\boldsymbol{x}, \boldsymbol{\gamma}),
\end{array}\right.
$$

where $x_{\mathrm{E}}$ and $z_{\mathrm{E}}$ correspond to a given location of the physical space as commonly used in an Eulerian description. Algebraically speaking, $\Psi(\boldsymbol{x}, \boldsymbol{\gamma})$ plays the role of a Hamiltonian function if $x$ and $z$ are considered as playing the role of conjugate variables. ${ }^{24}$ This analogy is purely functional (dynamical). The stream function $\Psi$ is a constant of motion that preserves the volume in the state space (in the Liouville sense) as the energy does in a conservative system. Thus, we will show that, when the $m$ Galerkin modes are governed by a conservative system (2), the system is actually conservative. 
The nonautonomous dynamical system (1) can be rewritten in the $(m+2)$-dimensional autonomous form

$$
\left\{\begin{array}{l}
\dot{\gamma}=f(\gamma) \\
\dot{x}=V(x, \gamma)
\end{array}\right.
$$

An integration of this system produces the trajectory in $\mathbb{R}^{2}(\boldsymbol{x})$. The particle is selected by determining the initial condition $x(0)$. A given evolution of the velocity field $\boldsymbol{V}(\boldsymbol{x}, \boldsymbol{\gamma})$ for the particles of a given flow is determined by choosing a set of initial conditions $\gamma(0)$. Notice that the Lagrangian description is provided by the whole system (5).

An Eulerian description consists in looking at fluid particles through a given location $\boldsymbol{x}_{\mathrm{E}} \subset \mathbb{R}^{2}(\boldsymbol{x}){ }^{1,25}$ The two components of the velocity field are obtained according to

$$
\left\{\begin{array}{l}
\dot{\gamma}=f(\gamma) \\
V_{\mathrm{E}}(t)=\nabla \wedge \Psi\left(x_{\mathrm{E}}, \gamma\right)
\end{array}\right.
$$

The Eulerian description is, therefore, independent from the spatial dynamics in $\mathbb{R}^{2}(\boldsymbol{x})$. The Eulerian description only contains information about the dynamics governing the velocity field $V(x, \gamma)$ without any knowledge about the geometry of the flow.

The presence of a chaotic time-dependence of the velocity field (Eulerian chaos) is not required for Lagrangian chaos to occur, as it is well-known. ${ }^{3,4,26,27}$ Since the spatial dynamics is driven by the subsystem (2), it is not possible to get a nonchaotic motion in $\mathbb{R}^{2}(\boldsymbol{x})$ with a chaotic velocity time-dependence.

It is important to distinguish the notion of trajectory $\mathscr{T} \subset \mathbb{R}^{2}(\boldsymbol{x})$ made of the successive locations visited by a fluid particle from the solution $\mathscr{S}(t) \subset \mathbb{R}^{m+2}(\boldsymbol{x}, \boldsymbol{\gamma})$ of the system (5): $\mathscr{T}$ is a bidimensional projection of the solution $\mathscr{S}$. As we will see, $\mathscr{T}$ can be a closed orbit even when the solution $\mathscr{S}(t)$ is chaotic (Table I).

Investigating the autonomous dynamical system (5), it is now possible to propose some definitions as follows.

Definition 1. Eulerian chaos: When there is a chaotic timedependence for the velocity field $\boldsymbol{V}(\boldsymbol{x}, \boldsymbol{\gamma})$, that is, when $\mathscr{V} \subset \mathbb{R}^{m}(\gamma)$ is governed by a chaotic dynamics, then we say that there is Eulerian chaos.

Remark 1. Eulerian chaos can be obtained if and only if $m \geq 3$.

Definition 2. Lagrangian chaos: When the solution $\mathscr{S} \subset \mathbb{R}^{m+2}$ $(\boldsymbol{x}, \boldsymbol{\gamma})$ to the whole system (5) is chaotic, then there is Lagrangian chaos.

Remark 2. Due to the Poincaré-Bendixson theorem, Lagrangian chaos can be obtained if and only if $m \geq 1$. Since a periodic solution is at least required for the mode dynamics (2), in practice, Lagrangian chaos can be obtained if $m>1$.

TABLE I. Different types of solution $\mathscr{S}$ depending on the nature of the time-dependence $\mathscr{V}$ of the velocity $\mathbf{V}$. The cases of the Rayleigh-Bénard (RB) convection and of the driven double-gyre (DG) are also reported.

\begin{tabular}{cccc}
\hline \hline $\mathscr{V} \subset \mathbb{R}^{m}(\boldsymbol{\gamma})$ & Constant & Periodic & Chaotic \\
$\mathscr{S} \subset \mathbb{R}^{m+2}(\boldsymbol{x}, \boldsymbol{\gamma})$ & Constant & Periodic & Chaotic \\
& Periodic & $\begin{array}{c}\text { Quasiperiodic } \\
\text { Chaotic }\end{array}$ & \\
Example & & DG $(m=2)$ & RB $(m=3)$ \\
\hline \hline
\end{tabular}

Remark 3. The chaotic nature of the solutions $\mathscr{V}$ and $\mathscr{S}$ can be determined using the techniques borrowed to the nonlinear dynamical systems theory ${ }^{28,29}$ by working in the space $\mathbb{R}^{m}(\boldsymbol{\gamma})$ and $\mathbb{R}^{m+2}(\boldsymbol{x}, \boldsymbol{\gamma})$, respectively.

Definition 3. Chaotic mixing: Let us consider a velocity field whose asymptotic evolution is determined by the initial conditions $\gamma(0)$ at time $t_{0}$ in a given Poincaré section $\mathscr{P}$. Let a chaotic solution be $\mathscr{S} \subset \mathbb{R}^{m+2}(\boldsymbol{x}, \boldsymbol{\gamma})$ and $\mathscr{D}_{c} \subset \mathbb{R}^{2}(\boldsymbol{x})$ the domain of the Poincaré section visited by the solution $\mathscr{S}$ such that the volume $\operatorname{Vol}\left(\mathscr{D}_{c}\right) \neq 0$. Let $\mathscr{N}_{0} \subset \mathscr{D}_{c}$, a neighborhood filled at $t_{0}$ with particles of a tracer and let $\boldsymbol{x} \in \mathscr{D}_{c}, \mathscr{N}_{x} \subset \mathscr{D}_{c}$, a neighborhood of the same volume as $\mathscr{N}_{0}$. Let $P\left(\mathscr{N}_{x}\right)$ be the probability of finding particles of the tracer in $\mathscr{N}_{x}$. There is chaotic mixing if $\forall x \in \mathscr{D}_{c}$,

$$
\lim _{t \rightarrow \infty} P\left(\mathscr{N}_{x}\right) \approx \frac{\operatorname{Vol}\left(\mathscr{N}_{0}\right)}{\operatorname{Vol}\left(\mathscr{D}_{c}\right)}
$$

Remark 4. A chaotic solution $\mathscr{S} \subset \mathbb{R}^{5}(\boldsymbol{x}, \boldsymbol{\gamma})$ can be associated with a closed trajectory $\mathscr{T} \subset \mathbb{R}^{2}(\boldsymbol{x})$ as well as exemplified in the fivedimensional Lorenz system (see Sec. III). In that case, $\operatorname{Vol}\left(\mathscr{D}_{c}\right)=0$ and there is no chaotic mixing.

Remark 5. Chaotic mixing requires Lagrangian chaos but Lagrangian chaos does not necessarily imply chaotic mixing.

\section{SIMPLIFIED RAYLEIGH-BÉNARD FLOW}

The classical two-dimensional Rayleigh-Bénard convection in which cells have a length along the $y$-axis larger than their heights along the $z$-axis is considered here. The velocity field was derived in the Boussinesq approximation..$^{30,31}$ The widths and the heights of the cells are adimensionalized in such a way that $0<x<\pi$ and $0<z<\pi$, respectively. There is a rotation symmetry by $\pi$ between two adjacent cells, leading to a clockwise convection for one cell and a counterclockwise one for the other. The 1963 Lorenz model is a low-dimensional truncation of the partial differential equations

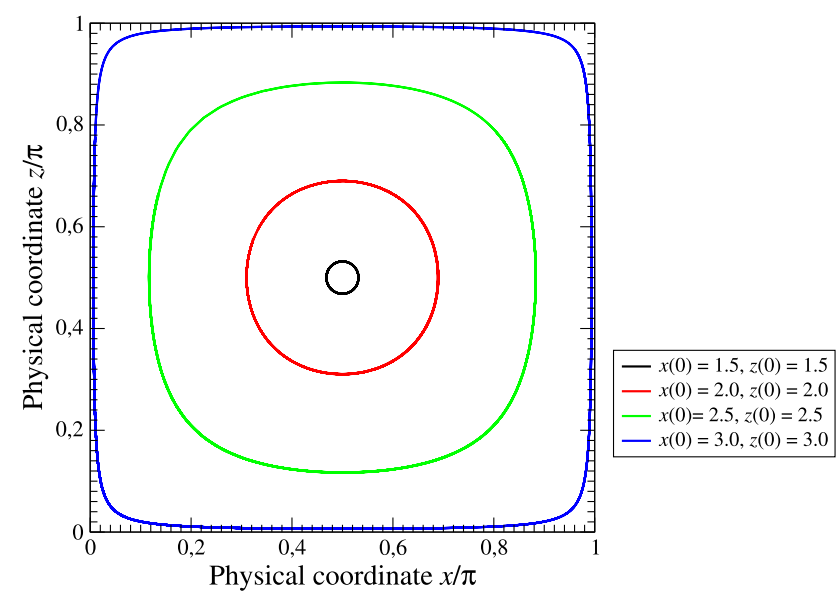

FIG. 1. Trajectories of the fluid particles in the physical space spanned by variables $x$ and $z$ for a few different sets of coordinates. Parameter values: $R=28, \sigma=10, b=\frac{8}{3}$, and $A=\sqrt{2}$. Other initial conditions: $\gamma_{1}(0)=0.1$, $\gamma_{2}(0)=0.5$, and $\gamma_{3}(0)=0.1$. 
describing the velocity field of the Rayleigh-Bénard problem. The three variables spanning the mode space are $\gamma_{1}$ for the intensity of the convection motion, $\gamma_{2}$ for the difference in the temperature between the left and the right sides of a convection roll, and $\gamma_{3}$ for the difference from the linear profile of temperature observed without convection. ${ }^{32}$ The 1963 Lorenz model governs the time-dependence of the velocity field $\boldsymbol{V}(\boldsymbol{x}, \boldsymbol{\gamma})$. The three variables $\gamma_{i}(i=1,2,3)$ are related to the stream function $\Psi$ and the temperature gradient $\delta T$ as $^{33}$

$$
\mid \begin{aligned}
& \Psi=A \gamma_{1} \sin (x) \sin (z), \\
& \delta T=A \gamma_{2} \cos (x) \sin (z)-\gamma_{3} \sin (2 z) .
\end{aligned}
$$

Using the relationships between the stream function $\Psi$ and the velocity $\boldsymbol{V}$, the Lagrangian form of the truncated Rayleigh-Bénard system can be written as the five-dimensional autonomous system,

$$
\left\{\begin{array}{l}
\dot{\gamma}_{1}=\sigma\left(\gamma_{2}-\gamma_{1}\right) \\
\dot{\gamma}_{2}=R \gamma_{1}-\gamma_{2}-\gamma_{1} \gamma_{3} \\
\dot{\gamma}_{3}=-b \gamma_{3}+\gamma_{1} \gamma_{2} \\
\dot{x}=A \gamma_{1} \sin (x) \cos (z) \\
\dot{z}=-A \gamma_{1} \cos (x) \sin (z)
\end{array}\right.
$$

where $A=\sqrt{2} ; R, \sigma$, and $b$ are the parameters of the 1963 Lorenz system. ${ }^{13}$ The three-dimensional mode dynamics drives the two-dimensional spatial dynamics. The state space associated with this five-dimensional Lorenz system is spanned by the vector $\boldsymbol{\Gamma}=\left(x, z, \gamma_{1}, \gamma_{2}, \gamma_{3}\right)=(\boldsymbol{x}, \boldsymbol{\gamma}) \in \mathbb{R}^{5}$.

The Jacobian matrix of the system (9) reads as
The trace of this Jacobian matrix is

$$
\operatorname{Tr}(\mathscr{J})=-(\sigma+1+b)+A \gamma_{1} \cos (x) \cos (z) \underbrace{(1-1)}_{=0} .
$$

The spatial dynamics governing the trajectories of the fluid particles is, therefore, conservative, while the three-dimensional mode dynamics is dissipative. The entire system should be considered as semidissipative (or semiconservative).

If one sets $\boldsymbol{x}=\boldsymbol{x}_{\mathrm{E}}$ for the location of an Eulerian description, one gets a time series, which is equivalent (modulo a similitude) to the mode $\gamma_{1}$ of the 1963 Lorenz system (according to the first equation of [8)]: the solution $\mathscr{V}\left(\boldsymbol{x}_{\mathrm{E}}, \boldsymbol{\gamma}\right)$ to the dynamics underlying the Eulerian description is, therefore, chaotic. When the motion of a given fluid particle is investigated in the space $\mathbb{R}^{2}(x, z)$, the trajectory $\mathscr{T}$ is a closed curve (Fig. 1). Nevertheless, since $\mathscr{V}$ is chaotic, $\mathscr{S}$ is necessarily chaotic too.

When a state portrait is reconstructed from the variable $x$ using derivative coordinates, ${ }^{34,35}$ the chaotic nature is unveiled (Fig. 2). In fact, a continuum of chaotic attractors is observed: such a property is specific to semidissipative systems. ${ }^{5}$ A fluid particle flows along a closed trajectory $\mathscr{T}$, which is visited in a chaotic way: the velocity is chaotically modulated. For each closed trajectory $\mathscr{T}$ in the space $\mathbb{R}^{2}(\boldsymbol{x})$, there is one chaotic attractor in $\mathbb{R}^{5}(\boldsymbol{x}, \boldsymbol{\gamma})$ : one may switch from one attractor to the other only by changing the initial conditions $x(0)$ and $z(0)$, as shown in Fig. 2. The motion of fluid particles is, therefore, chaotic and there is Lagrangian chaos, but there is no chaotic mixing since $\mathscr{D}_{\mathrm{c}}=0$ in the Poincaré section projected in the physical space,

$$
P_{\mathrm{L}} \equiv\left\{\left(x_{n}, z_{n}\right) \in \mathbb{R}^{2} \mid \gamma_{3, n}=R-1, \dot{\gamma}_{3, n}>0\right\} .
$$

An introduction to observability analysis can be found in the Appendix. Let us now perform this analysis on the five-dimensional system (9). Since the mode dynamics drives the spatial component, measuring only $\gamma_{i}(i=1,2,3)$ would lead to a null observability of $\mathbb{R}^{5}(\boldsymbol{x}, \boldsymbol{\gamma})$. Contrary to this, one could expect quite a good observability when the variables $x$ and $z$ are measured. From these measurements, two possible reconstructed spaces are spanned by

$$
\Phi_{x^{3} z^{2}}=\mid \begin{aligned}
& X_{1}=x, \\
& X_{2}=\dot{x}, \\
& X_{3}=\ddot{x}, \\
& X_{4}=z, \\
& X_{5}=\dot{z},
\end{aligned} \quad \text { and } \quad \Phi_{x^{2} z^{3}}=\mid \begin{aligned}
& X_{1}=x, \\
& X_{2}=\dot{x}, \\
& X_{3}=z, \\
& X_{4}=\dot{z}, \\
& X_{5}=\ddot{z} .
\end{aligned}
$$

where, for instance, the state vector $\boldsymbol{X}=x^{3} z^{2}=(x, \dot{x}, \ddot{x}, z, \dot{z})$. The Jacobian matrix $\mathscr{J}_{\Phi_{x^{3} z^{2}}}$ of this coordinate transformation corresponds to the observability matrix. ${ }^{11}$ A full observability of the original state space from $x$ and $z$ would mean that any pair of different states such that $\Gamma_{1} \neq \Gamma_{2}$ in the original state space are distinguished in the reconstructed space, that is, $\boldsymbol{X}_{1} \neq \boldsymbol{X}_{2}$. A full observability corresponds to a full rank observability matrix or, equivalently, to Det $\mathscr{J}_{\Phi_{x^{3} z^{2}}} \neq 0$ for any state. For instance, the Jacobian matrix

$$
\mathscr{J}_{\Phi_{x^{3} z^{2}}}=\left[\begin{array}{ccccc}
0 & 0 & 0 & 1 & 0 \\
A \sin (x) \cos (z) & 0 & 0 & A \gamma_{1} \cos (x) \cos (z) & -A \gamma_{1} \sin (x) \sin (z) \\
A \sin (x)\left[2 A \gamma_{1} \cos (x)-\sigma \cos (z)\right] & A \sigma \sin (x) \cos (z) & 0 & A \sigma\left(\gamma_{2}-\gamma_{1}\right) \cos (x) \cos (z)+A^{2} \gamma_{1}^{2} \cos (2 x) & -A \sigma\left(\gamma_{2}-\gamma_{1}\right) \sin (x) \sin (z) \\
0 & 0 & 0 & 0 & 1 \\
-A \cos (x) \sin (z) & 0 & 0 & A \gamma_{1} \sin (x) \sin (z) & -A \gamma_{1} \cos (x) \cos (z)
\end{array}\right]
$$



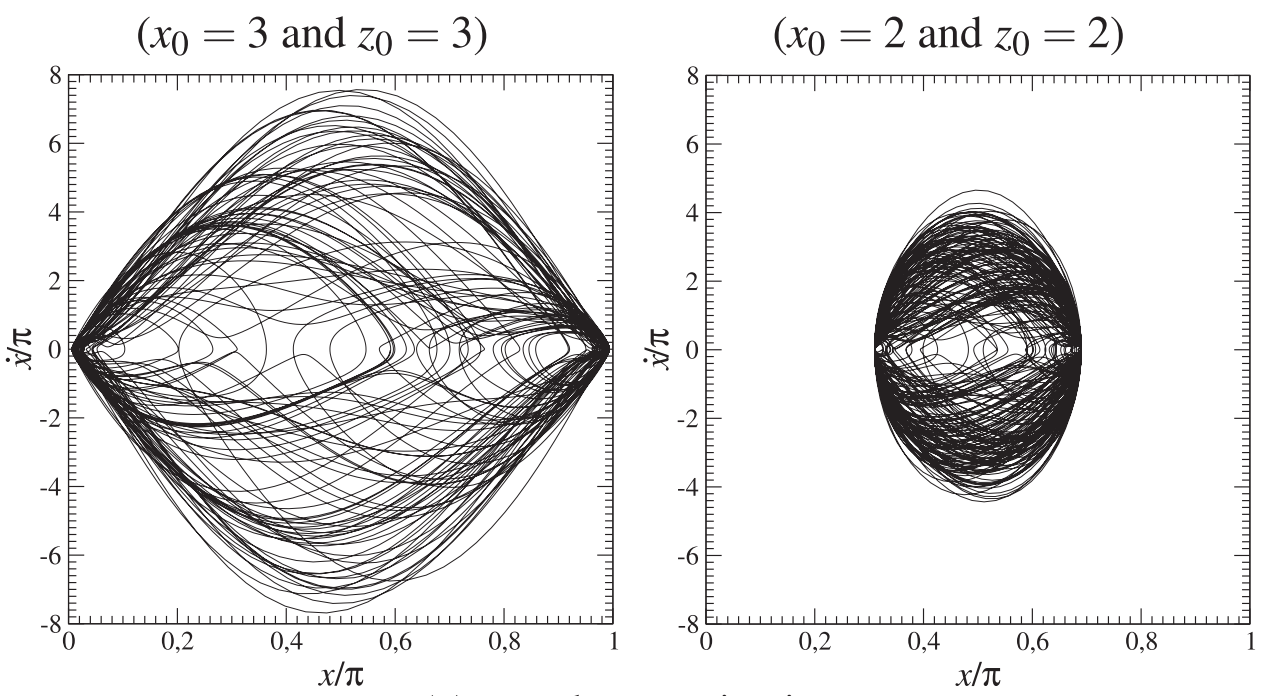

(a) $x-\dot{x}$ plane projection
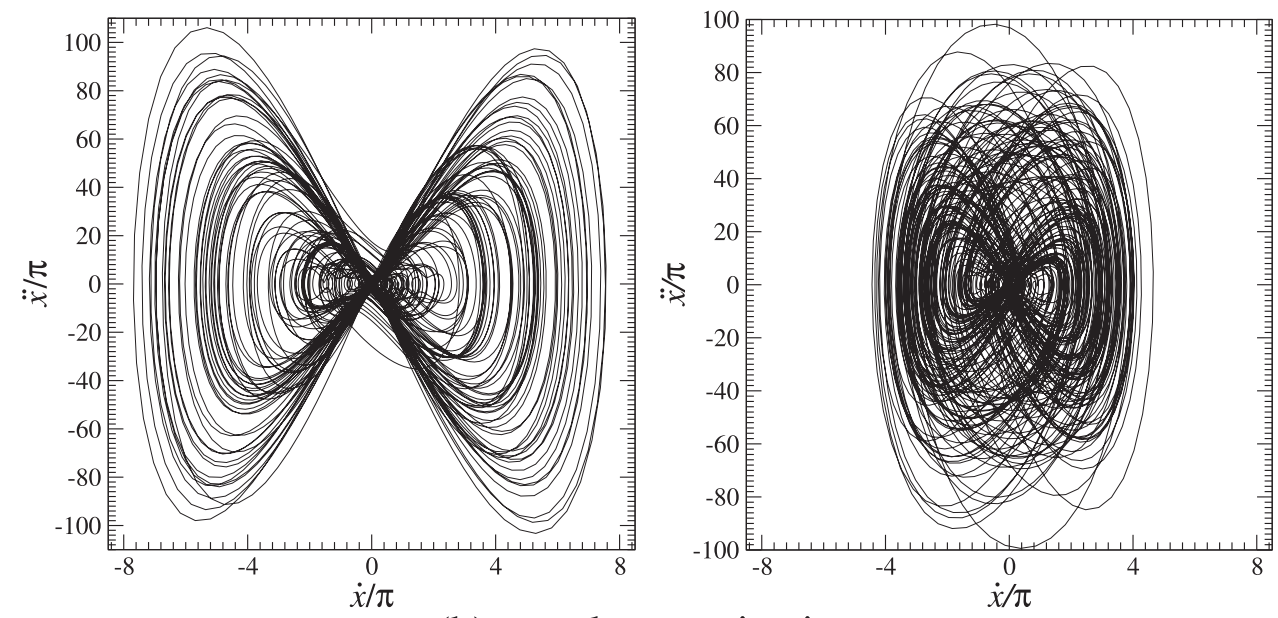

FIG. 2. Trajectories of two different fluid particles (two different sets of initial conditions) in two plane projections of the state space reconstructed from variable $x$ by using derivative coordinates. Parameter values and other initial conditions as in has a null determinant: this means that the five-dimensional Lorenz dynamics cannot be adequately observed in the physical subspace from the trajectory of one fluid particle in $\mathbb{R}^{2}(\boldsymbol{x})$. Note that none of the variables offers a good observability alone of the original state space $\mathbb{R}^{5}(\boldsymbol{x}, \boldsymbol{\gamma})$. Variables $\gamma_{i}(i=1,2,3)$ are necessarily associated with a null observability of the state space since they do not have information about the spatial variables. As explained in the Appendix, a symbolic observability coefficient $\delta$ greater than 0.75 means that the observability is sufficiently good for a reliable analysis. ${ }^{36}$ The two spatial variables have a very poor observability since their associated symbolic observability coefficients are $\delta_{x^{5}}=\delta_{z^{5}}=0.06$, where $x^{5}$ means that the vector used to reconstruct the phase space is made of the five Lie derivatives of $x$, that is, of variable $x$ and its first four time derivatives (see Ref. 37 for more details). It is necessary to measure at least three variables for providing a good observability: for instance, the space spanned by
$\boldsymbol{X}=\left(\gamma_{1}, \dot{\gamma}_{1}, \gamma_{3}, x, \dot{x}\right)$ is associated with a symbolic observability coefficient $\delta_{\gamma_{1}^{2} \gamma_{3} x^{2}}=0.84$. It is indeed required to measure some variables of the subspace $\mathbb{R}^{3}\left(\gamma_{1}, \gamma_{2}, \gamma_{3}\right)$ to perform a reliable analysis of the dynamics.

In the Eulerian approach, a position $\boldsymbol{x}_{\mathrm{E}}=\left(x_{\mathrm{E}}, z_{\mathrm{E}}\right)$ is chosen and the second equation of the system (5) is no longer meaningful. In the case of the five-dimensional Lorenz model (9), the Eulerian velocity is governed by the system

$$
\left\{\begin{array}{l}
V_{x_{\mathrm{E}}}=A \gamma_{1}(t) \sin \left(x_{\mathrm{E}}\right) \cos \left(z_{\mathrm{E}}\right), \\
V_{z_{\mathrm{E}}}=-A \gamma_{1}(t) \cos \left(x_{\mathrm{E}}\right) \sin \left(z_{\mathrm{E}}\right),
\end{array}\right.
$$

where $\gamma_{1}(t)$ is solution to the three-dimensional Lorenz subsystem. There is no analytical expression for $\gamma_{1}(t)$ since the behavior is chaotic for the chosen parameter values. It clearly appears that the 
Eulerian velocity is then governed by the three-dimensional Lorenz dynamics.

\section{THE DRIVEN DOUBLE-GYRE FLOW}

Let us consider the driven double-gyre flow corresponding to two adjacent convection cells as in the Rayleigh-Bénard convection but in which a lateral oscillation

$$
g\left(x, \gamma_{1}\right)=\gamma_{1}(x-2)
$$

of the roll pattern is inserted. Contrary to the light perturbation inserted by Solomon and Gollub, ${ }^{38}$ here this is a strong perturbation that is applied. ${ }^{18}$ The stream function of the driven double-gyre system is given by

$$
\Psi\left(x, z, \gamma_{1}\right)=A \sin \left(\pi x\left[\left(1+g\left(x, \gamma_{1}\right)\right]\right) \sin (\pi z) .\right.
$$

It defines a cellular flow with boundaries free of shear stresses. Due to the quadratic term $\gamma_{1} x^{2}$ introduced by perturbation (14), the natural symmetry observed between two adjacent convection cells is broken in this double-gyre system. To restore the natural symmetry associated with a Rayleigh-Bénard convection, we derived a simple fully symmetrized variant of the equations that govern the driven double-gyre flow in Sec. IV B.

Here, the perturbation corresponds to a periodic forcing applied to the velocity field. This forcing term is responsible for the diffusion of fluid particles from one cell to the other. This model is a simplification of a double-gyre pattern that occurs frequently in geophysical flows. ${ }^{18,39,40}$

The driving term $\gamma_{1}=\eta \sin (\omega t)$ is the solution to the secondorder differential equation

$$
\ddot{\gamma}_{1}+\omega^{2} \gamma_{1}=0
$$

where the amplitude $\eta$ is defined by the initial conditions

$$
\mid \begin{aligned}
& \gamma_{1}(0)=0, \\
& \gamma_{2}(0)=\omega \eta .
\end{aligned}
$$

This is a one degree of freedom oscillator whose energy is constant. Since a plane is required for drawing a period-1 orbit, the two dimensions must be kept for the analysis.

From the stream function $\Psi\left(x, z, \gamma_{1}\right)$, the governing equations are defined according to Eq. (4); combined with the driving process (16), we get the four-dimensional dynamical system

$$
\left\{\begin{array}{l}
\dot{\gamma}_{1}=\gamma_{2} \\
\dot{\gamma_{2}}=-\omega^{2} \gamma_{1} \\
\dot{x}=-A \pi \sin \left(\pi x\left[1+\gamma_{1}(x-2)\right]\right) \cos (\pi z) \\
\dot{z}=A \pi\left(2 \gamma_{1}[x-1]+1\right) \\
\quad \times \cos \left(\pi x\left[1+\gamma_{1}(x-2)\right]\right) \sin (\pi z)
\end{array}\right.
$$

where the driving term $\gamma_{1}$ is explicitly described. System (18) corresponds to the driven double-gyre rewritten in an autonomous form. The corresponding state space is thus spanned by the four variables $x, z, \gamma_{1}$, and $\gamma_{2}$. Notice that the velocity of the fluid particle does no longer contribute to determining its state as previously seen. The trace of the Jacobian of system (18) is null: the fluid particles are, therefore, governed by a four-dimensional autonomous conservative system.
The time-dependence of the velocity field is purely periodic as characterized by the differential equation (16). The special way the perturbation function $g\left(x, \gamma_{1}\right)$ is constructed is responsible for the chaotic mixing. When the perturbation is removed $(\eta=0)$, the fluid particles describe closed trajectories that are visited periodically. When $\eta>0$, the trajectories are no longer closed and correspond to chaotic motions when the fluid particles visit the neighborhood of the boundary between two adjacent cells; the motion is quasiperiodic otherwise.

\section{A. Dynamical analysis}

A numerical integration of system (18) from appropriate initial conditions leads to the chaotic behavior shown in Fig. 3.

The flow is developed within the intervals $x \in[0,2]$ and $z \in[0,1]$. It is possible to center the flow in order to simplify the governing equations without changing the dynamics. This is obtained by applying the coordinate transformation $(x, z) \mapsto\left(X+1, Z+\frac{1}{2}\right)$, leading to

$$
\left\{\begin{array}{l}
\dot{\gamma}_{1}=\gamma_{2}, \\
\dot{\gamma}_{2}=-\omega^{2} \gamma_{1}, \\
\dot{X}=-A \pi \sin \left(\pi\left[\gamma_{1} X^{2}+X-\gamma_{1}\right]\right) \sin (\pi Z), \\
\dot{Z}=-A \pi\left(2 \gamma_{1} X+1\right) \cos \left(\pi\left[\gamma_{1} X^{2}+X-\gamma_{1}\right]\right) \cos (\pi Z) .
\end{array}\right.
$$

The trajectory is now bounded by the $2 \times 1$ rectangle centered around the origin of the state space $\mathbb{R}^{4}(\boldsymbol{X}, \boldsymbol{\gamma})$. The singular points of system (19) are defined by

$$
\mid \begin{aligned}
& \gamma_{1}=0, \\
& \gamma_{2}=0, \\
& \sin (\pi X) \sin (\pi Z)=0, \\
& \cos (\pi X) \cos (\pi Z)=0,
\end{aligned}
$$

leading to

$$
\boldsymbol{X}_{1}=\mid \begin{aligned}
& X_{1}=k, \\
& Z_{1}= \pm \frac{k}{2},
\end{aligned} \quad \text { and } \quad X_{2}=\mid \begin{aligned}
& X_{2}= \pm \frac{k}{2}, \\
& Z_{2}=0,
\end{aligned}
$$

where $k \in \mathbb{N}$. In the subspace $\mathbb{R}^{2}(\gamma)$, the eigenvalues are $\lambda_{3,4}= \pm i \omega$, thus defining a center in the $\gamma_{1}-\gamma_{2}$ plane. This is true for all the singular points. Points $\boldsymbol{X}_{1}$ are of the saddle-center type since associated with the eigenvalues $\lambda_{1,2}= \pm \pi^{2} A$ in the $X-Z$ plane. Points $\boldsymbol{X}_{2}$

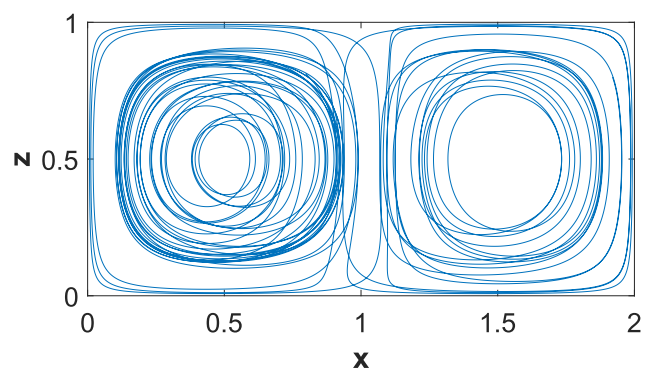

FIG. 3. Chaotic behavior produced by the driven double-gyre system (18). Parameter values: $A=0.1$, and $\omega=\frac{\pi}{5}$. Initial conditions: $x(0)=1, z(0)=0.5$, $\gamma_{1}(0)=0$, and $\gamma_{2}(0)=0.0628009$. 


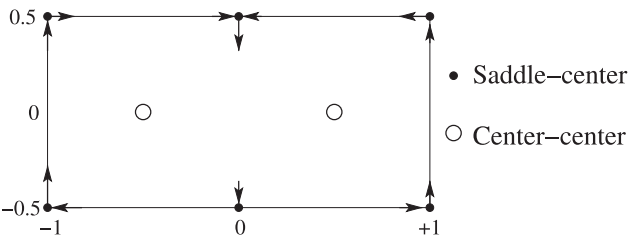

FIG. 4. Locations of the eight singular points in the $X-Z$ plane.

are of the center-center type since associated with the eigenvalues $\lambda_{1,2}= \pm i \pi^{2} A$.

The state space is, therefore, bounded as drawn in Fig. 4. This four-dimensional autonomous conservative system is investigated using the Poincaré section

$$
\mathscr{P} \equiv\left\{\left(X_{n}, Z_{n}, \gamma_{2, n}\right) \in \mathbb{R}^{3} \mid \gamma_{1, n}=0, \gamma_{2, n}>0\right\} .
$$

Since the system is conservative, the behavior strongly depends on the initial conditions. As commonly observed in conservative systems ${ }^{41}$ there is a chaotic sea surrounding regular islands which are centered around

$$
\boldsymbol{X}_{\mathrm{A}}=\mid \begin{aligned}
& X_{\mathrm{A}}= \pm 0.59, \\
& Z_{\mathrm{A}}=\mp 0.38,
\end{aligned} \quad \text { and } \quad \boldsymbol{X}_{\mathrm{B}}=\mid \begin{aligned}
& X_{\mathrm{B}}= \pm 0.48, \\
& Z_{\mathrm{B}}=\mp 0.08,
\end{aligned}
$$

that is, around period-1 points (Fig. 5). In the Poincaré section, since $\gamma_{1}=0$, there is a central symmetry with respect to the origin of the state space. It is, therefore, possible to obtain the symmetric of a given

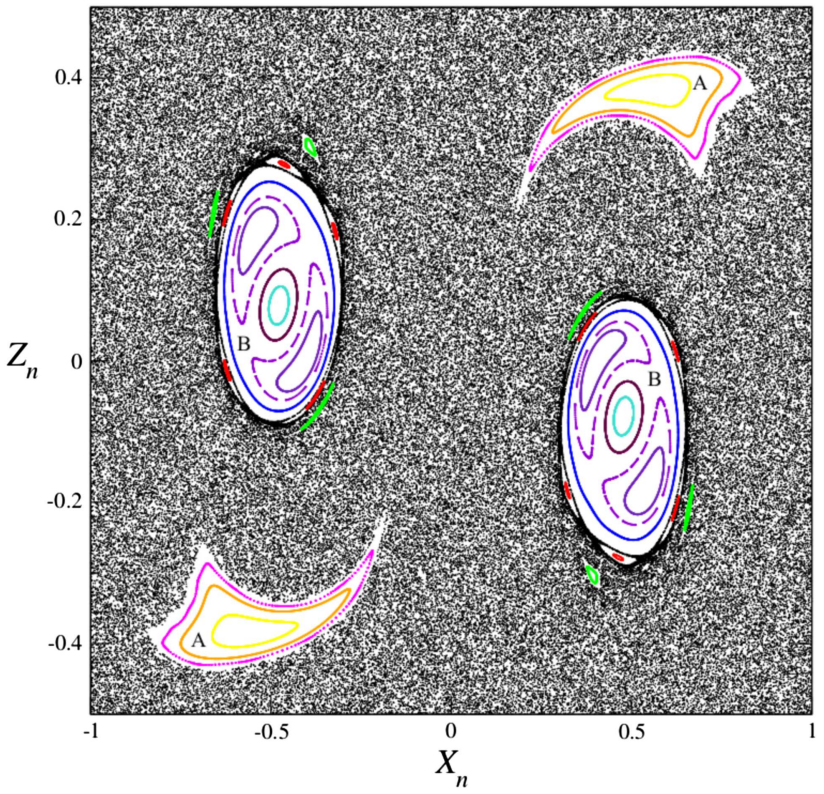

FIG. 5. Poincare section of the centered driven double-gyre system (19) for various initial conditions. A chaotic sea surrounds the symmetry-related regular islands $A$ and the symmetry-related regular islands $B$. Parameter values: $A=0.1$, and $\omega=\frac{\pi}{5}$. Other initial conditions: $\gamma_{1}(0)=0$ and $\gamma_{2}(0)=0.0628009$. island by applying the matrix

$$
\mathscr{I}=\left[\begin{array}{cccc}
-1 & 0 & 0 & 0 \\
0 & -1 & 0 & 0 \\
0 & 0 & -1 & 0 \\
0 & 0 & 0 & -1
\end{array}\right],
$$

which defines the inversion symmetry with respect to origin of the state space.

The resulting Poincaré section is shown for various initial conditions in Fig. 5. In islands A, there are only period-1 tori whose Poincaré sections are characterized by a single annular structure. Two of them are shown in Fig. 6(a). One is the symmetric of the other, at least in the Poincaré section. In islands B, it is possible to observe tori with two annular structures in the Poincaré section; the trajectory visits the second structure before returning to the first one. Two such tori are shown in Fig. 6(b). Around islands B, there are period-3 tori

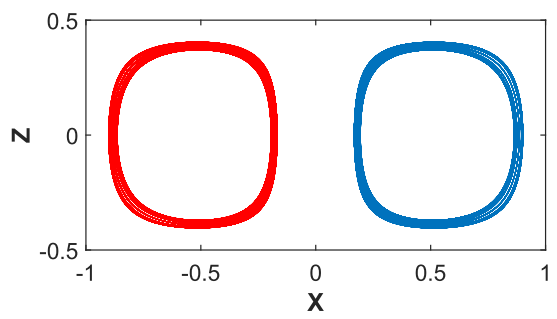

(a) Period-1 tori from islands $\mathrm{A}$

$X(0)= \pm 0.6$ and $Z(0)= \pm 0.4, \gamma_{1}(0)=0$, and $\gamma_{2}(0)=0.0628009$

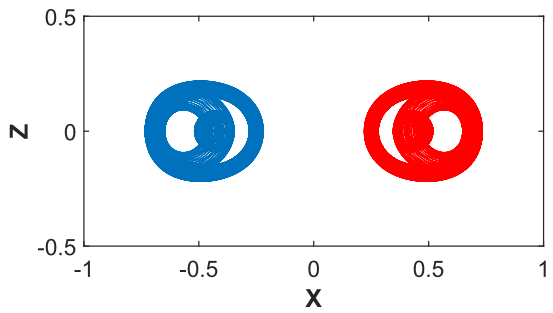

(b) Period-2 tori from islands $\mathrm{B}$

$X(0)= \pm 0.58, Z(0)= \pm 0.12, \gamma_{1}(0)=0$, and $\gamma_{2}(0)=0.0628009$

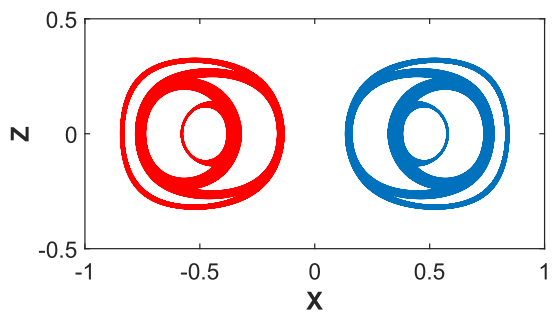

(c) Period-3 tori surrounding islands A $X(0)= \pm 0.405, Z(0)= \pm 0.315, \gamma_{1}(0)=0$, and $\gamma_{2}(0)=0.0628009$

FIG. 6. Examples of quasiperiodic motions produced by the driven double-gyre system (19). Same parameter values as in Fig. 5. 
(in green in Fig. 5): the trajectories visit the two other annular structures once before returning to the first one [Fig. 6(c)]. Around islands $B$, there are also islands of period- 5 tori (not shown), surrounded by the chaotic sea, with only a very tiny chaotic band separating these two types of islands.

The state space associated with the driven double-gyre system (19) is spanned by the state vector $\boldsymbol{\Gamma}=(\boldsymbol{X}, \boldsymbol{\gamma})$. For assessing the observability of the state space, the symbolic observability coefficients are computed from the symbolic Jacobian matrix

$$
\mathscr{J}_{\mathrm{DG}}^{\mathrm{ym}}=\left[\begin{array}{cccc}
\overline{1} & \overline{1} & \overline{1} & 0 \\
\overline{1} & \overline{1} & \overline{1} & 0 \\
0 & 0 & 0 & 1 \\
0 & 0 & 1 & 0
\end{array}\right],
$$

where 1 designates a constant element $J_{i j}$ of the Jacobian matrix of system (19) and $\overline{1}$ a nonconstant element. When the reconstructed vector is made of the four Lie derivatives of $X$, the symbolic observability coefficient is $\delta_{X^{4}}=0.17$. An equal symbolic observability coefficient is found when the variable $Z$ is the only measured variable. The determinant of both observability matrices $\mathscr{O}_{X^{4}}$ and $\mathscr{O}_{Z^{4}}$ is very complicated, thus confirming the poor observability of the original state space when either $X$ or $Z$ is the sole measured variable.

An additional insight is provided when a differential embedding is constructed by using the Lie derivatives of the measured variable. The space reconstructed from the variable $X$ presents an inversion symmetry, thus allowing to easily distinguish the left from the right sides [Fig. 7(a)]. Such a possibility is not provided by the variable $Z$, which leads to a single "leaf" attractor [Fig. 7(b)]. It is known that an observability analysis is not sensitive to symmetry property. ${ }^{43}$

When a Poincaré section is defined as

$$
\mathscr{P}_{s}=\left\{\left(s_{n}, \dot{s}_{n}, \ddot{s}_{n}\right) \in \mathbb{R}^{3} \mid \dot{s}_{n}=0, \ddot{s}_{n}>0\right\},
$$

where $s=X$ or $s=Z$, then islands observed in the original state space (Fig. 5) are not detected because the regular islands are only well-identified in a Poincaré section taking into account the period of the driving term. This shows how sensitive to the choice of measurements are some specific dynamical properties of Lagrangian chaos. Notice that measuring simultaneously the two variables $X$ and $Z$ does not improve the situation since the corresponding symbolic observability matrix

$$
\mathscr{O}_{X^{2} Z^{2}}=\left[\begin{array}{cccc}
1 & 0 & 0 & 0 \\
\overline{1} & \overline{1} & \overline{1} & 0 \\
0 & 1 & 0 & 0 \\
\overline{1} & \overline{1} & \overline{1} & 0
\end{array}\right]
$$

is rank deficient: the original state space is not observable when variables $X$ and $Z$ are simultaneously measured.

Contrary to this, the situation is significantly improved when the driving term $\gamma_{1}$ is also measured. This is, for instance, confirmed by the symbolic observability coefficients $\delta_{X^{2} \gamma_{1}^{2}}=\delta_{Z^{2} \gamma_{1}^{2}}=0.81$ meaning there is a good observability of the state space. The measurement of the driving term is particularly useful for correctly defining the Poincaré section as

$$
\mathscr{P}=\left\{\left(s_{n}, \dot{s}_{n}, \dot{\gamma}_{1, n}\right) \in \mathbb{R}^{3} \mid \gamma_{1, n}=0, \dot{\gamma}_{1, n}>0\right\},
$$

where $s=X$ or $s=Z$. When $s=X$, the largest regular island is easily detected [bottom part of the Poincaré section, Fig. 8(a)]. The fact that the variable $Z$ does not allow to discriminate easily the left and the right sides of the convection cell blurs the islands by superimposing them to the chaotic sea [Fig. 8(b)]. Consequently, when the driven double-gyre system is investigated, it is more reliable to measure the variables $X$ and $\gamma_{1}$ than $Z$ and $\gamma_{1}$ and definitely better than $X$ and $Z$. We thus recover the general result obtained with the Lorenz system: Lagrangian chaos is correctly investigated when the analysis is performed in the entire state space $\mathbb{R}^{m+2}(\boldsymbol{X}, \boldsymbol{\gamma})$ and not only in the space $\mathbb{R}^{2}(\boldsymbol{X})$. (a) From variable $X$

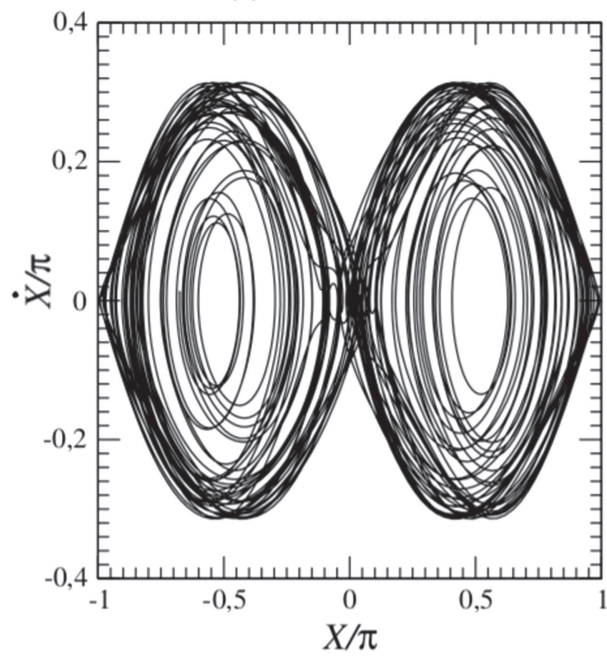

(b) From variable $Z$

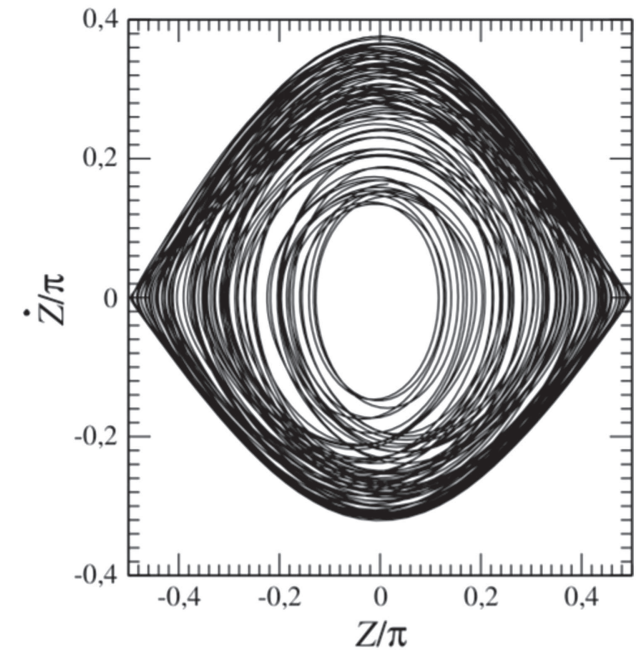

FIG. 7. Differential embedding induced by the variable $X$ (a) and the variable $Z$ (b). Parameter values as in Fig. 5. 
(a) $X$-induced differential embedding

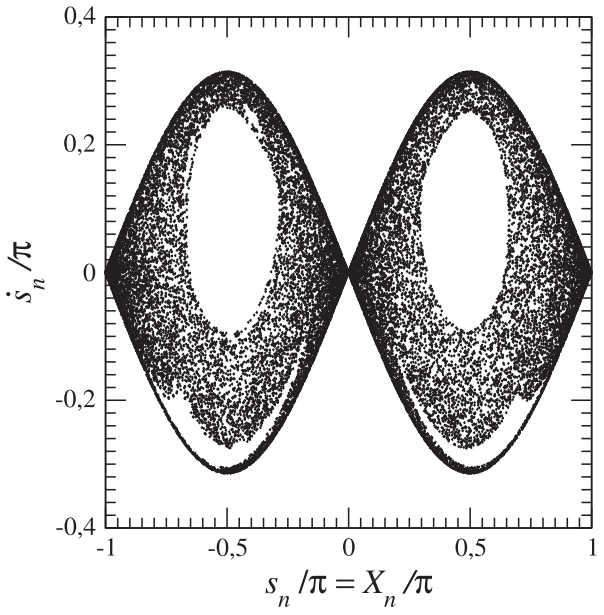

(b) Z-induced differential embedding

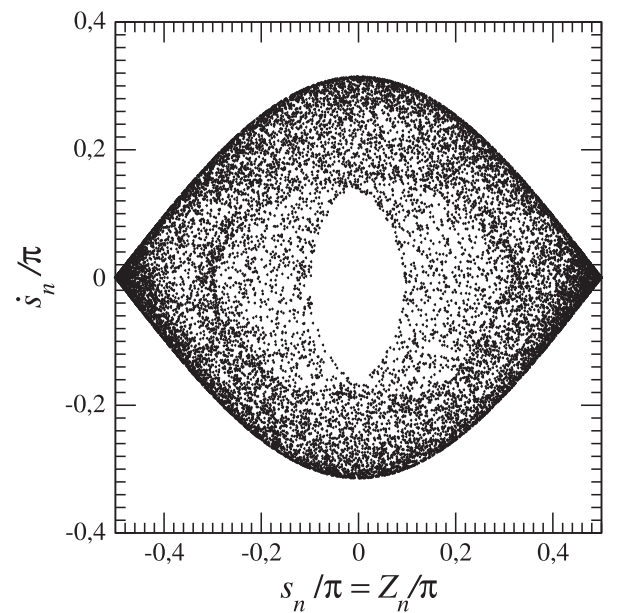

FIG. 8. Poincare section $\mathscr{P}$ for the differential embedding induced by the variable $X$ (a) and the variable $Z$ (b). Parameter values as in Fig. 5.

\section{B. A simplified symmetric variant of the driven double-gyre flow}

In the original driven double-gyre system (18), the symmetry is only observed in the Poincaré section since the condition $\gamma_{1}=0$ is required to cancel the term $2 \gamma_{1} X$ in the fourth equation of system (19): this term breaks the inversion symmetry that one could expect when flow cells are identical. It is possible to restore the symmetry by removing this term, which is not necessary for getting a doublegyre-like dynamics. Moreover, one can further simplify the system

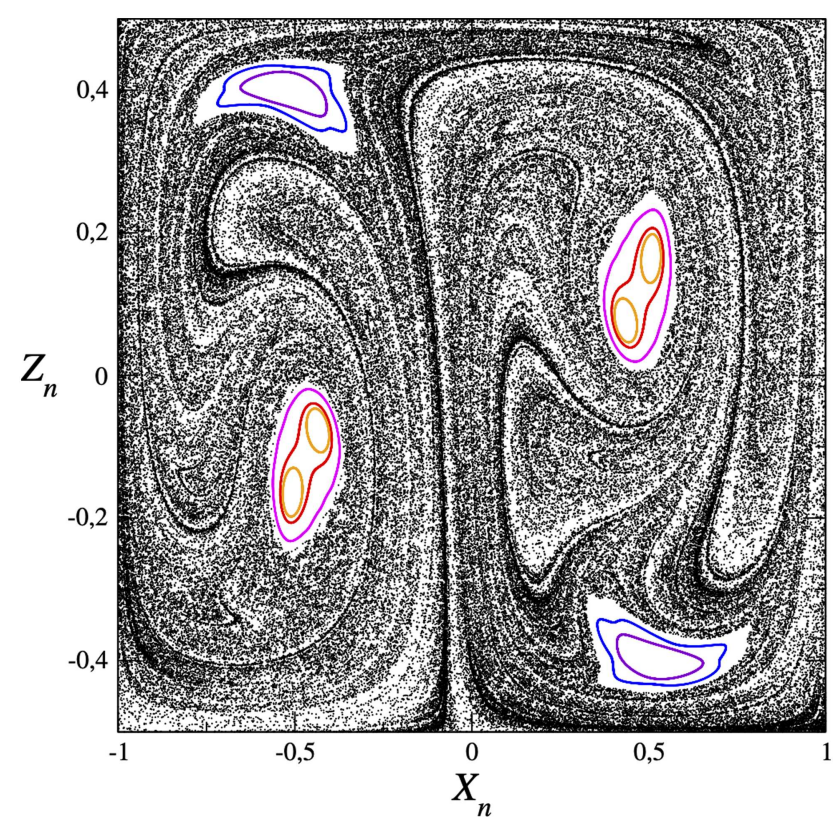

FIG. 9. Poincaré section of the symmetrized driven double-gyre system (29) for various initial conditions. Parameter values: $A=0.14$ and $\omega=\frac{\pi}{5}$. by removing the minus signs from the right hand sides of $\dot{X}$ and $\dot{Z}$ equations, since they do not significantly alter the dynamics. This leads to

$$
\left\{\begin{array}{l}
\dot{\gamma}_{1}=\gamma_{2}, \\
\dot{\gamma}_{2}=-\omega^{2} \gamma_{1}, \\
\dot{X}=A \pi \sin \left(\pi\left[\gamma_{1} X^{2}+X-\gamma_{1}\right]\right) \sin (\pi Z), \\
\dot{Z}=A \pi \cos \left(\pi\left[\gamma_{1} X^{2}+X-\gamma_{1}\right]\right) \cos (\pi Z) .
\end{array}\right.
$$

This system is equivariant under an inversion symmetry with respect to the origin of the state space, that is, it obeys ${ }^{42}$

$$
\boldsymbol{G}(\mathscr{I} \cdot \Gamma)=\mathscr{I} \cdot \boldsymbol{G}(\boldsymbol{\Gamma}),
$$

where $\boldsymbol{G}$ is the vector field associated with the system (29). By slightly modifying the parameter value $\eta$, it is possible to obtain a rather similar state portrait as revealed by the Poincaré section (Fig. 9). Restoring the inversion symmetry presents the advantage of having simpler equations and a flow whose stretching-and-folding structure is better detected.

\section{CONCLUSION}

This work considers the role of observability in laminar bidimensional fluid flows expressed as autonomous dynamical systems. General considerations are provided leading to definitions of Lagrangian and Eulerian chaos in this perspective.

It is shown that in order to correctly determine whether the dynamics is chaotic or not, the whole corresponding state space must be used. The Eulerian description is independent from the Lagrangian. The dynamics underlying the time-dependence of the velocity field can be investigated without any ambiguity in the space spanned by all the modes resulting from a Galerkin technique. Consequently, the tools borrowed from the nonlinear dynamical systems theory can be used to determine the nature of the dynamics underlying the velocity field. In contrast, the characterization of the Lagrangian dynamics suffers from the customary use of governing equations written in a nonautonomous form, that is, from working in a projection of the full state space. In particular, the corresponding 
state subspace associated with the $m$ modes retained in a Galerkin technique is not explicitly defined. Rewritten under autonomous form, the Lagrangian system is forced by the mode(s) driving the dynamics, and the state space required for investigating the motion of fluid particles is the whole $(m+2)$-dimensional state space. In that case, Lagrangian chaos presents the properties commonly considered in the nonlinear dynamical systems theory.

In the autonomous formulation, the Lagrangian dynamics of two-dimensional incompressible flows always presents a twodimensional subsystem that is driven by the time-dependence of the velocity field. When this time-dependence is chaotic, the Lagrangian dynamics is necessarily chaotic. This time-dependence can be either dissipative or conservative. In the former case, the system is semidissipative (or semiconservative) and there is a continuum of attractors. In the latter case, there is neither an attractor nor a transient regime. Using the whole state space, we showed that, indeed, a periodic mode dynamics can lead to Lagrangian chaos. When the Lagrangian dynamics is investigated in the whole state space, all the results are in agreement with the nonlinear dynamical systems theory but may contradict the literature analyzing Lagrangian chaos in physical space. We suggested considering that Lagrangian chaos corresponds to a chaotic solution in the whole state space. It is thus possible that such a chaotic solution is associated with a projection in physical space, which is a closed orbit visited in a chaotic way. In that case, there is no mixing.

The importance of adopting this frame in order to achieve a reliable characterization of the fluid dynamics is illustrated with two simple models of incompressible flows. The existence of chaotic solutions with a closed-orbit projection in physical space is well exemplified with the five-dimensional Lorenz system. We showed that the driven double-gyre system can be rewritten as an autonomous four-dimensional conservative system. A symmetrized version of the driven double-gyre is also presented. According to the observability analysis of these flows, it appears that the dynamics underlying fluid particles is poorly characterized when the trajectory in the physical space is only used. If only the spatial coordinates are measured, an embedding-using for instance delay or derivative coordinates-with a sufficiently large dimension should be used for a nonambiguous characterization.

\section{ACKNOWLEDGMENTS}

This work is supported by the MATH-GEO (18-MATH-04) project of the Regional Program MATH-AmSud. G.D.C. acknowledges support of her Ph.D. thesis by CONICET. C.L. thanks Irène Sendiña Nadal for having computed the symbolic observability coefficients.

\section{APPENDIX: A BRIEF INTRODUCTION TO OBSERVABILITY}

In control theory, there is a concept to assess the quality of information that is conveyed by some measurements. It is based on the observability matrix ${ }^{10}$ that is defined as the Jacobian matrix of the coordinate transformation $\Phi: \mathbb{R}^{d}(\boldsymbol{y}) \mapsto \mathbb{R}^{d}(\boldsymbol{Y})$ between the original state space $\mathbb{R}^{d}(\boldsymbol{y})$ and the space $\mathbb{R}^{d}(\boldsymbol{Y})$ reconstructed from the measured variables and some of the Lie derivatives. ${ }^{11}$
Let us start by considering the $d$-dynamical system

$$
\dot{x}_{i}=f_{i}(\boldsymbol{x}),
$$

where $\boldsymbol{x} \in \mathbb{R}^{d}$ is the state vector and $f_{i}$ is the $i$ th component of the vector field $\boldsymbol{f}$. The dynamical system (A1) is said to be state observable at time $t$ if the initial state $\boldsymbol{x}(0)$ can be uniquely determined from the knowledge of $m$ measured variables $\boldsymbol{h}(\boldsymbol{x}) \in \mathbb{R}^{m}(m<d)$ and some of their derivatives in the interval $[0, t],{ }^{44}$ thus forming a vector $\boldsymbol{X} \in \mathbb{R}^{d_{\mathrm{r}}}$ composed of the variables spanning the reconstructed space. Practically, the observability of system (A1) through the reconstructed vector $\boldsymbol{X}$ is assessed by computing the rank of the observability matrix

$$
\mathscr{O}_{\boldsymbol{X}}(\boldsymbol{x})=\left[\begin{array}{c}
\mathrm{d} h_{1}(\boldsymbol{x}) \\
\mathrm{d} \mathscr{L}_{\boldsymbol{f}} h_{1}(\boldsymbol{x}) \\
\vdots \\
\mathrm{d} h_{2}(\boldsymbol{x}) \\
\mathrm{d} \mathscr{L}_{f} h_{2}(\boldsymbol{x}) \\
\vdots \\
\mathrm{d} h_{m}(\boldsymbol{x}) \\
\mathrm{d} \mathscr{L}_{\boldsymbol{f}} h_{m}(\boldsymbol{x}) \\
\vdots
\end{array}\right],
$$

where $\mathrm{d} \equiv \frac{\partial}{\partial x}$ and $\mathscr{L}_{f}^{k} h_{i}(\boldsymbol{x})$ is the $k$ th Lie derivative of the $i$ th component $h_{i}(\boldsymbol{x})$ of measurements along the vector field $\boldsymbol{f} .{ }^{10}$ This observability matrix corresponds to the Jacobian matrix of the coordinate transformation $\Phi_{X}$ between the original state space $\mathbb{R}^{d}$ and the reconstructed space $\mathbb{R}^{d_{\mathrm{r}}}{ }^{11}$ System (A1) is said to be state observable if and only if the observability matrix has full rank, that is, $\operatorname{rank}\left(\mathscr{O}_{\mathbf{X}}\right)=d$. An equivalent way to assess the observability is to compute the determinant of the observability matrix $\mathscr{O}_{X}$ and to check that it never vanishes. It was shown that the more complex the determinant, the smaller the observability.

We would like to avoid a yes-or-no answer since a system may gradually become nonobservable when a parameter is varied. In order to do that, the degree of observability can be quantified by using the observability coefficient $\delta^{\prime}(\boldsymbol{x}),{ }^{45,46}$

$$
\delta^{\prime}(\boldsymbol{x})=\frac{\left|\lambda_{\min }\left[\mathscr{O} \mathscr{O}^{\mathrm{T}}(\boldsymbol{x}, t)\right]\right|}{\left|\lambda_{\max }\left[\mathscr{O} \mathscr{O}^{\mathrm{T}}(\boldsymbol{x}, t)\right]\right|} .
$$

The numerator of this coefficient corresponds to the smallest eigenvalue of $\mathscr{O} \mathscr{O}^{\mathrm{T}}$ estimated at point $\boldsymbol{x}$, while the denominator corresponds to the largest eigenvalue. It is convenient to use the mean value $\overline{\delta^{\prime}(\boldsymbol{x})}$ along a trajectory $\{\boldsymbol{x}\}$ to assess the observability of a given dynamics. The coefficient $\delta^{\prime}(\boldsymbol{x}) \in[0,1]: 0$ (1) corresponds to a non (fully) observable state space through the measurements. In spite of many trials, it was not possible to normalize these coefficients to have them equal to one in the case of full observability. This was one of the motivations for introducing the symbolic observability coefficients. ${ }^{47}$ These fractional observability coefficients quantify the dynamical observability.

Another inconvenience of these fractional observability coefficients is that they do not allow to compare the observability of different systems. Moreover, they cannot be computed for systems whose dimension is too large. The symbolic observability coefficients were then introduced. ${ }^{37,47,49}$ They can be computed as follows. 
First, the Jacobian matrix $\mathscr{J}$ is transformed into a symbolic Jacobian matrix $\tilde{\mathscr{J}}$ by replacing each constant element $J_{i j}$ by 1 , each polynomial element $J_{i j}$ by $\overline{1}$, and each rational element $J_{i j}$ by $\overline{\overline{1}}$ when the $j$ th variable is present in the denominator or by $\overline{1}$ otherwise. Rational terms are distinguished from polynomial terms since they reduce the observability more significantly than the latter ones. ${ }^{49}$

Second, for the sake of simplicity, let us consider a univariate measurement $s=h(\boldsymbol{x})=x_{i}$. Thus, the first row of $\tilde{\mathscr{O}}_{s}$ is just defined by the derivative of the measurement function $\mathrm{d} h$, that is, $\tilde{O}_{1 i}=1$ if the $i$ th variable is measured and 0 otherwise. The second row is directly obtained from $\tilde{\mathcal{J}}$ by copying its $i$ th row, that is, $\tilde{O}_{2 j}=\tilde{J}_{i j} \forall j$, being $i$ the index of the measured variable. The $k$ th row is obtained as follows. Each element $\tilde{\mathscr{J}}_{i j}$ of the $i$ th row of $\tilde{\mathscr{J}}$ is multiplied by the corresponding $i$ th component of the vector $v=\left(\tilde{O}_{\ell 1}, \ldots, \tilde{O}_{\ell d}\right)$ where $\ell=k-1$ refers to the $(k-1)$ th row of the symbolic observability matrix $\tilde{\mathscr{O}}_{s}$. The rules to perform the symbolic product $\tilde{J}_{i j} \otimes v_{i}$ are such that $^{49}$

$$
\mid \begin{aligned}
& 0 \otimes a=0, \\
& 1 \otimes a=a, \\
& \overline{1} \otimes a=a \text { for } a=\overline{1}, \overline{\overline{1}}, \\
& \overline{\overline{1}} \otimes a=\overline{\overline{1}} \text { for } a \neq 0 .
\end{aligned}
$$

Then, the resulting symbolic Jacobian matrix $\tilde{J}^{\prime}$ is thus reduced into a row where each element $\tilde{O}_{k j}=\sum_{i} \tilde{J}_{i j}^{\prime}$ is just the sum of the elements of the $j$ th column according to the addition law ${ }^{49}$

$$
\mid \begin{aligned}
& 0 \oplus a=a, \\
& 1 \oplus a=a \text { for } a \neq 0, \\
& \overline{1} \oplus a=a \text { for } a \neq 0,1, \\
& \overline{\overline{1} \oplus a=\overline{\overline{1}} .}
\end{aligned}
$$

When $m$ variables are measured, $\tilde{\mathscr{O}}_{s}$ is constructed by blocks of size $\left(d_{i}+1\right) \times d, d_{i}$ being the number of derivatives of $s_{i}$ and $\sum_{i=1}^{m} d_{i}$ $+m=d$. Each block is constructed according to the previous rules.

Third, the determinant of $\tilde{\mathscr{O}}_{s}$ is computed according to the symbolic product rule defined in (A4) and expressed as products and addends of the symbolic terms $1, \overline{1}$, and $\overline{\overline{1}}$, whose number of occurrences are $N_{1}, N_{\overline{1}}$ and $N_{\overline{1}}$, respectively. A special condition is required for rational systems such that if $N_{\overline{1}}=0$ and $N_{\overline{1}} \neq 0$, then $N_{\overline{1}}=N_{\overline{1}}$. The symbolic observability coefficient for the measurement $s$ is equal to

$$
\delta_{s}=\frac{1}{D} N_{1}+\frac{1}{D^{2}} N_{\overline{1}}+\frac{1}{D^{3}} N_{\overline{1}},
$$

with $D=\max \left(1, N_{1}\right)+N_{\overline{1}}+N_{\overline{1}}$ and $0 \leq \delta_{s} \leq 1$, where $\delta_{s}=1$ for a combination providing full observability.

These coefficients are within the unit interval, 0 being associated with a null observability and one with a full observability. The observability of a system via some measurements is good when the symbolic observability coefficient is beyond 0.75 . $^{36}$

\section{REFERENCES}

${ }^{1}$ G. K. Batchelor, An Introduction to Fluid Dynamics (Cambridge University Press, 2000).

${ }^{2}$ S. Wiggins and J. M. Ottino, "Foundations of chaotic mixing," Philos. Trans. R. Soc. London 362, 937-970 (2004).
${ }^{3}$ J. M. Ottino, "Mixing, chaotic advection and turbulence," Annu. Rev. Fluid Mech. 22, 207-253 (1990).

${ }^{4}$ T. Bohr, M. H. Jensen, G. Paladin, and A. Vulpiani, Dynamical Systems Approach to Turbulence (Cambridge University Press, 2005).

${ }^{5}$ O. Ménard, C. Letellier, J. Maquet, L. L. Sceller, and G. Gouesbet, "Analysis of a non-synchronized sinusoidally driven dynamical system," Int. J. Bifurcat. Chaos 10, 1759-1772 (2000).

${ }^{6}$ A. Carrassi, M. Ghil, A. Trevisan, and F. Uboldi, "Data assimilation as a nonlinear dynamical systems problem: Stability and convergence of the predictionassimilation system," Chaos 18, 023112 (2008).

${ }^{7} \mathrm{M}$. Ghil, "The compatible balancing approach to initialization, and fourdimensional data assimilation," Tellus 32, 198-206 (1980).

${ }^{8} \mathrm{M}$. Ghil and P. Malanotte-Rizzoli, Data Assimilation in Meteorology and Oceanography (Elsevier, 1991), pp. 141-266.

${ }^{9}$ M. Ghil, "Advances in sequential estimation for atmospheric and oceanic flows," J. Meteorolog. Soc. Japan Ser. II 75, 289-304 (1997).

${ }^{10}$ R. Hermann and A. Krener, "Nonlinear controllability and observability," IEEE Trans. Automat. Contr. 22, 728-740 (1977).

${ }^{11}$ C. Letellier, L. A. Aguirre, and J. Maquet, "Relation between observability and differential embeddings for nonlinear dynamics," Phys. Rev. E 71, 066213 (2005).

${ }^{12}$ P. Bergé, Y. Pomeau, and C. Vidal, L'ordre dans le Chaos-Vers une Approche Déterministe de la Turbulence (Hermann, 1997).

${ }^{13}$ E. N. Lorenz, "Deterministic nonperiodic flow," J. Atmos. Sci. 20, 130-141 (1963).

${ }^{14} \mathrm{H}$. Yang and Z. Liu, "Chaotic transport in a double gyre ocean," Geophys. Res. Lett. 21, 545-548, https://doi.org/10.1029/94gl0030 (1994).

${ }^{15}$ A. C. Poje and G. Haller, "Geometry of cross-stream mixing in a double-gyre ocean model," J. Phys. Oceanogr. 29, 1649-1665 (1999).

${ }^{16}$ E. Simonnet, M. Ghil, and H. Dijkstra, "Homoclinic bifurcations in the quasigeostrophic double-gyre circulation,” J. Marine Res. 63, 931-956 (2005).

${ }^{17}$ T. Matsuura and M. Fujita, "Two different aperiodic phases of wind-driven ocean circulation in a double-gyre, two-layer shallow-water model," J. Phys. Oceanogr. 36, 1265-1286 (2006).

${ }^{18}$ S. C. Shadden, F. Lekien, and J. E. Marsden, "Definition and properties of Lagrangian coherent structures from finite-time Lyapunov exponents in twodimensional aperiodic flows," Physica D 212, 271-304 (2005).

${ }^{19}$ K. G. D. S. Priyankara, S. Balasuriya, and E. Bollt, "Quantifying the role of folding in nonautonomous flows: The unsteady double-gyre," Int. J. Bifurcat. Chaos 27, 1750156 (2017).

${ }^{20} \mathrm{D}$. Lipinski and $\mathrm{K}$. Mohseni, "A ridge tracking algorithm and error estimate for efficient computation of Lagrangian coherent structures," Chaos 20, 017504 (2010).

${ }^{21}$ M. R. Allshouse and T. Peacock, "Lagrangian based methods for coherent structure detection," Chaos 25, 097617 (2015).

${ }^{22}$ K. R. Pratt, J. D. Meiss, and J. P. Crimaldi, "Reaction enhancement of initially distant scalars by Lagrangian coherent structures," Phys. Fluids 27, 035106 (2015).

${ }^{23}$ M. O. Williams, I. I. Rypina, and C. W. Rowley, "Identifying finite-time coherent sets from limited quantities of Lagrangian data," Chaos 25, 087408 (2015).

${ }^{24}$ H. Aref, J. R. Blake, M. Budišić, S. S. S. Cardoso, J. H. E. Cartwright, H. J. H. Clercx, K. El Omari, U. Feudel, R. Golestanian, E. Gouillart, G. F. van Heijst, T. S. Krasnopolskaya, Y. Le Guer, R. S. MacKay, V. V. Meleshko, G. Metcalfe, I. Mezić, A. P. S. de Moura, O. Piro, M. F. M. Speetjens, R. Sturman, J.-L. Thiffeault, and I. Tuval, "Frontiers of chaotic advection," Rev. Mod. Phys. 89, 025007 (2017).

${ }^{25}$ H. Lamb, Hydrodynamics (Cambridge University Press, 1994).

${ }^{26} \mathrm{M}$. Hénon, "Sur la topologie des lignes de courant dans un cas particulier," C. R. Acad. Sci. 262, 312-314 (1966)

${ }^{27} \mathrm{H}$. Aref, "Stirring by chaotic advection," J. Fluid Mech. 143, 1-21 (1984).

${ }^{28} \mathrm{H}$. Kantz and T. Schreiber, Nonlinear Time Series Analysis (Cambridge University Press, 2010)

${ }^{29} \mathrm{R}$. Gilmore and M. Lefranc, The Topology of Chaos (Wiley, 2003).

${ }^{30} \mathrm{H}$. Jeffreys, "The stability of a layer of fluid heated below," Philos. Mag. VII $\mathbf{2}$, 833-844 (1926). 
${ }^{31}$ S. Chandrasekhar, Hydrodynamics and Hydromagnetic Stability (Dover, New York, 1961).

${ }^{32}$ C. Letellier, P. Dutertre, and G. Gouesbet, "Characterization of the Lorenz system, taking into account the equivariance of the vector field," Phys. Rev. E 49, 3492-3495 (1994).

${ }^{33}$ G. P. Massimo Falcioni and A. Vulpiani, "Regular and chaotic motion of fluid particles in a two-dimensional fluid," J. Phys. A 21, 3451-3462 (1988).

${ }^{34}$ N. H. Packard, J. P. Crutchfield, J. D. Farmer, and R. S. Shaw, "Geometry from a time series," Phys. Rev. Lett. 45, 712-716 (1980).

${ }^{35}$ F. Takens, "Detecting strange attractors in turbulence," Lect. Notes Math. 898, 366-381 (1981).

${ }^{36}$ I. Sendiña Nadal, S. Boccaletti, and C. Letellier, "Observability coefficients for predicting the class of synchronizability from the algebraic structure of the local oscillators," Phys. Rev. E 94, 042205 (2016).

${ }^{37}$ C. Letellier, I. Sendiña-Nadal, E. Bianco-Martinez, and M. S. Baptista, "A symbolic network-based nonlinear theory for dynamical systems observability," Sci. Rep. 8, 3785 (2018).

${ }^{38}$ T. H. Solomon and J. P. Gollub, "Chaotic particle transport in timedependent Rayleigh-Bénard convection," Phys. Rev. A 38, 6280-6286 (1988).

${ }^{39} \mathrm{C}$. Couliette and S. Wiggins, "Intergyre transport in a wind-driven, quasigeostrophic double gyre: An application of lobe dynamics," Nonlinear Process. Geophys. 7, 59-85 (2000).
${ }^{40} \mathrm{H}$. A. Dijkstra and M. Ghil, "Low-frequency variability of the large-scale ocean circulation: A dynamical systems approach," Rev. Geophys. 43, RG3002, https://doi.org/10.1029/2002RG000122 (2005).

${ }^{41}$ S. Wiggins, Introduction to Applied Nonlinear Dynamical Systems and Chaos (Springer-Verlag, New York, 2003).

${ }^{42} \mathrm{C}$. Letellier and R. Gilmore, "Covering dynamical systems: Twofold covers," Phys. Rev. E 63, 016206 (2000).

${ }^{43} \mathrm{C}$. Letellier and L. A. Aguirre, "Investigating nonlinear dynamics from time series: The influence of symmetries and the choice of observables," Chaos 12, 549-558 (2002).

${ }^{44} \mathrm{~T}$. Kailath, Linear Systems, Information and System Sciences Series (PrenticeHall, 1980).

${ }^{45} \mathrm{~L}$. A. Aguirre, "Controllability and observability of linear systems: Some noninvariant aspects," IEEE Trans. Educ. 38, 33-39 (1995).

${ }^{46}$ C. Letellier, J. Maquet, L. L. Sceller, G. Gouesbet, and L. A. Aguirre, "On the non-equivalence of observables in phase-space reconstructions from recorded time series," J. Phys. A 31, 7913-7927 (1998).

${ }^{47}$ C. Letellier and L. A. Aguirre, "Symbolic observability coefficients for univariate and multivariate analysis," Phys. Rev. E 79, 066210 (2009).

${ }^{48} \mathrm{~L}$. A. Aguirre, L. L. Portes, and C. Letellier, "Structural, dynamical and symbolic observability: From dynamical systems to networks," PLoS One 13, e0206180 (2018).

${ }^{49}$ E. Bianco-Martinez, M. S. Baptista, and C. Letellier, "Symbolic computations of nonlinear observability,” Phys. Rev. E 91, 062912 (2015). 